\title{
ЛАЗЕРНОЕ МИКРОСТРУКТУРИРОВАНИЕ ПОВЕРХНОСТИ ИЗДЕЛИЙ ИЗ МЕДИ
}

\author{
Е.О. Баранов ${ }^{1}$ В.Г. Бутенко ${ }^{2}$, О.И. Каширин ${ }^{1}$, П.О. Колосовский ${ }^{1}$, Ю.А. Кулагин ${ }^{1}$, М.С. Осипова ${ }^{2}$ \\ ${ }^{1}$ Государственный научный центр Российской Федерации Федеральное государственное унитарное \\ предприятие «Центральный научно-исследовательский институт химии и механики» (ГНЦ РФ ФГУП \\ «ЦНИИХМ»), ул. Нагатинская, д. 16а, г. Москва, Российская Федерация, 115487 \\ E-mail: mangst@yandex.ru,trg42ra@gmail.com, kolosovskypo@mail.ru, kulaginyua@mail.ru \\ ${ }^{2}$ Федеральное государственное бюджетное учреждение науки Межведомственный центр аналитических \\ исследований в области физики, химии и биологии при Президиуме Российской академии наук (МЦАИ \\ РАН), ул. Профсоюзная, 65, с.6, г. Москва, Российская Федерация, 117342 \\ E-mail: cnfc2009@mail.ru
}

Лазерное микро-наноструктурирование поверхности позволяет придавать материалам новые физические свойства: уменьшается трение в изделиях (до 70\%), происходит поверхностное термоупрочнение, увеличивается износостойкость и улучшаются скоростные характеристики изделий. Поэтому важно математическое прогнозирование характеристик профиля наносимых поверхностно-периодических структур (глубина и размеры лунок) для выбора режимов работы импульсного лазерного комплекса при изготовлении изделий с конкретной оптимальной микроструктурированной поверхностью. В статье рассмотрено использование закона Бугера-Ламберта-Бера для расчета геометрии профиля поверхностной микроструктуры, нанесенной одиночными лазерными импульсами на лабораторный образец из меди. В опубликованных материалах по теме микрострукттурирования поверхности импульсными лазерами приведены пороговые значения интенсивности лазерного излучения при которых происходит испарение материала лабораторного образца. Закон Бугера-Ламберта-Бера описывает ослабление интенсивности лазерного излучения при распространении его в поглощающей среде. Для обеспечения точности расчетов было произведено измерение средней мощности лазерного излучения и коэффициента отражения медного образца. На основании экспериментальных данных было получено значение показателя поглощения меди. Для проверки корректности расчетов на лабораторные образцы из меди лазером было нанесено два варианта микроструктуры. Сравнение результатов расчета геометрии профиля микроструктуры и результатов эксперимента показало наличие сходимости. Представленная методика может быть использована для прогнозирования профиля поверхностных микроструктур и для других материалов.

Ключевые слова: лазер, микроструктурирование, коэффициент отражения, показатель поглощения

\section{LASER MICROSTRUCTURING OF THE SURFACE OF COPPER PRODUCTS}

\author{
E.O. Baranov ${ }^{1}$, V.G. Butenko², O.I. Kashirin ${ }^{1}$, P.O. Kolosovskiy ${ }^{1}$, U.A. Kulagin ${ }^{1}$, M.S. Osipova ${ }^{2}$
}

${ }^{1}$ Central Research Institute of Chemistry and Mechanics, Nagatinskaya street, 16a, Moscow, Russian Federation, 115487

E-mail: mangst@yandex.ru,trg42ra@gmail.com, kolosovskypo@mail.ru, kulaginyua@mail.ru

${ }^{2}$ Federal State State-financed Institution of Sciences Interdepartmental Center of Analytic Studies in the Sphere of Physics, Chemistry and Biology under Presidium of Russian Academy of Sciences (ICAS RAS), 65 Trade Union Street, p. 6, Moscow, Russian Federation, 117342

E-mail: cnfc2009@mail.ru 
Laser micro-nanostructuring of the surface makes it possible to impart new physical properties to materials: friction in products decreases (up to 70\%), surface thermal hardening occurs, wear resistance increases and the speed characteristics of products improve. Therefore, it is important to mathematically predict the profile characteristics of the applied surface-periodic structures (depth and dimensions of the holes) for choosing the operating modes of the pulsed laser complex in the manufacture of products with a specific optimal microstructured surface. The article discusses the use of the Bouguer-Lambert-Beer law for calculating the geometry of the profile of the surface microstructure deposited by single laser pulses on a laboratory copper sample. In the published materials on the topic of surface microstructuring by pulsed lasers, the threshold values of the laser radiation intensity are given at which the material of the laboratory sample evaporates. The Bouguer-Lambert-Beer law describes the attenuation of the intensity of laser radiation when it propagates in an absorbing medium. To ensure the accuracy of the calculations, the average power of the laser radiation and the reflectance of the copper sample were measured. Based on the experimental data, the value of the absorption coefficient of copper was obtained. To check the correctness of the calculations, two variants of the microstructure were applied to laboratory copper samples by a laser. Comparison of the results of calculating the geometry of the profile of the microstructure and the results of the experiment showed the presence of convergence. The presented technique can be used to predict the profile of surface microstructures for other materials as well.

Key words: laser, microstructuring, reflection coefficient, absorption coefficient

Для цитирования:

Баранов Е.О., Бутенко В.Г., Каширин О.И., Колосовский П.О., Кулагин Ю.А., Осипова М.С. Лазерное микроструктурирование поверхности изделий из меди. Рос. хим. ж. (Ж. Рос. хим. об-ва). 2021. T. LXV. № 4. С. 65-71

For citation:

Baranov E.O., Butenko V.G., Kashirin O.I., Kolosovskiy P.O., Kulagin U.A., Osipova M.S. Laser microstructuring of the surface of copper products. Ros. Khim. Zh. 2021. V. 65. N 4. P. 65-71

Одним из направлений исследований в области новых материалов является создание на их поверхности микрорельефа. Наличие поверхностной микро-наноструктуры позволяет придавать материалам новые физические свойства. В проведенных нами ранее работах апробировалось нанесение периодических микро-наноструктур на различные изделия методом лазерной абляции с целью модификации их поверхности: лезвия коньков [1], поверхность пуль [2], дорожки качения подшипников [3]. Проведенная лазерная модификация поверхности позволила значительно уменьшить трение в изделиях (до 70\%), провести поверхностное термоупрочнение, увеличить износостойкость и улучшить скоростные характеристики изделий.

Метод лазерной абляции основан на воздействии импульсного лазерного излучения на поверхность материала, в следствии чего происходит быстрый нагрев поверхности. Тепло распространяется вглубь материала. При нагреве поверхности до температуры, превышающей температуру кипения материала, происходит испарение материала с образованием лунки. В процессе обработки различных материалов возникла необходимость математического прогнозирования характеристик профиля наносимых поверхностно-периодических структур (глубина и размеры лунок). Это важно для выбора режимов работы импульсного лазерного комплекса при изготовлении изделий с конкретной оптимальной микроструктурированной поверхностью. В [4] выведено соотношение, определяющее глубину лунки. Для единичного импульса оно имеет следующий вид:

$$
h=I \cdot \tau / L_{\mathrm{u}},
$$

где $I$ - интенсивность лазерного излучения на поверхности материала, $L_{\text {и }}$ - скрытая теплота испарения, $\tau$ - длительность лазерного импульса.

Однако, для задач расчета профиля наносимых микроструктур соотношение (1) не подходит, так как дает результаты, значительно отличающие от данных, полученных в экспериментальных исследованиях. В формуле присутствует параметр $L_{\text {и }}$ - скрытая теплота испарения, который известен не для всех материалов.

В этой работе в качестве одного из методов расчета геометрии профиля поверхностной микроструктуры предлагается использовать закон Бугера-Ламберта-Бера. Лазерное излучение, падающее на обрабатываемую поверхность, поглощается в соответствии с экспоненциальным законом [5].

$$
I(x)=I_{0}(1-R) e^{-\alpha x}
$$

где $I(x)$ - интенсивность лазерного излучения, 
проникшего в материал, $I_{0}-$ интенсивность лазерного излучения, падающего на обрабатываемую поверхность, $R$ - коэффициент отражения обрабатываемой поверхности, $\alpha$ - показатель поглощения обрабатываемой поверхности, $x$ - глубина проникания лазерного излучения.

Интенсивность лазерного излучения $I_{0}$, падающего на обрабатываемую поверхность определяется по формуле:

$$
I_{0}=\frac{P_{\text {имп }}}{S},
$$

где $P_{\text {имп }}-$ мощность импульса лазерного излучения, $\mathrm{S}$ - площадь пучка лазерного излучения в плоскости обработки.

Мощность лазерного импульса $P_{\text {имп }}$ определяется по формуле:

$$
P_{\text {имп }}=\frac{P}{f \cdot \tau},
$$

где $P$ - средняя мощность лазерного излучения, $f$ - частота лазерного излучения, $\tau$ - длительность лазерного импульса.

При определении средней мощности лазерного излучения Р необходимо учитывать ее зависимость от длительности импульса $\tau$ и частоты лазерного излучения $f$. Согласно ГОСТ 25786-83 [6] средняя мощность лазерного излучения определяется посредством прямого измерения мощности.

Частота лазерного излучения и длительность импульса зависят от используемого источника лазерного излучения. Площадь пятна лазерного излучения зависит от используемой сканаторной головки.

Закон Бугера (2) с учетом формул (3), (4) принимает вид:

$$
I(x)=\frac{4 \cdot P}{f \cdot \tau \cdot \pi \cdot d_{\Pi}^{2}}(1-R) e^{-\alpha x},
$$

где $d_{\text {п }}$ - диаметр лазерного пучка в плоскости обработки.

Для используемой в данной работе сканаторной головки диаметр пятна лазерного пучка в плоскости обработки составляет $d_{\Pi}=35$ мкм.

В [4] приведено соотношение для расчета пороговой интенсивности излучения лазерного импульса $I_{\text {имп }}^{\text {пор }}$ необходимой для нагрева металла до заданной температуры:

$$
I_{\text {имп }}^{\text {пор }}=\frac{\left(T-T_{\mathrm{H}}\right) \cdot k \sqrt{\pi}}{2(1-R) \sqrt{a \tau}}
$$

где $T$ - температура образца в зоне нагрева, $T_{\text {н }}$ начальная температура образца перед лазерной обработкой, $a$ - температуропроводность, $k$ - коэффициент теплопередачи.

Для определения пороговой интенсивности излучения лазерного импульса, необходимой для нагрева меди до температуры кипения, используем теплофизические параметры из табл. 1.
Таблица 1

Теплофизические параметры меди

\begin{tabular}{|c|c|}
\hline Параметр & Значение \\
\hline Температура испарения, К & 2840 \\
\hline Температура образца перед обработкой, К & 293 \\
\hline Коэффициент теплопередачи, Вт/К·м & 401 \\
\hline Температуропроводность, ${ }^{2} / \mathrm{c}$ & $0,12 \cdot 10^{-3}$ \\
\hline
\end{tabular}

Формула (6), при подстановке значений из таблицы 1 , позволяет проводить расчет пороговой интенсивности излучения лазерного импульса заданной длительностью $\tau$, необходимой для нагрева меди до температуры кипения:

$$
I_{\text {имп }}^{\text {пор }}=\frac{8,263 \cdot 10^{7}}{(1-R) \sqrt{\tau}}
$$

Длительность воздействия лазерного импульса на обрабатываемую поверхность составляет $\leq 200$ нс, площадь лазерного пучка в плоскости обработки $\pi \cdot \frac{d_{\text {п }}^{2}}{4}=\pi \frac{35^{2}}{4}=962$ мкм $^{2}$, при облучении поверхности лазером с пороговой интенсивностью излучения необходимой для нагрева меди до температуры кипения, принимается допущение о мгновенном испарении металла при $I(x) \geq I_{\text {имп }}^{\text {пор }}$. Соответственно, при $I(x)=I_{\text {имп }}^{\text {пор }, ~ п р о и з о и ̆ д е т ~ и с п а-~}$ рение металла на глубину $x$. Принятое допущение позволяет получить из формулы (5) зависимость глубины $x$ профиля поверхностных микроструктур от параметров материала и лазерного излучения:

$$
x=-\frac{\ln \frac{2,07 \cdot 10^{7} \cdot f \cdot \sqrt{\tau} \cdot \pi \cdot d_{\Pi}^{2}}{P \cdot(1-R)^{2}}}{\alpha}
$$

Формула (8) справедлива для единичных импульсов.

С целью проведения расчета глубины профиля поверхностных микроструктур, наносимых импульсным лазером необходимо определить параметры лазерного излучения и оптические характеристики медных образцов в формуле (8). В таблице 2 описан метод определения каждого параметра.

В соответствии с табл. 2, параметры №1, 5, 6 определяется экспериментально. Параметры №2-4 предоставляются производителем, либо задаются программно через устройство управления лазерного комплекса. При проведении экспериментов использовался лазерный комплекс LDesigner co следующими характеристиками [7]: длина волны излучения $\lambda=1,06$ мкм, средняя мощность излучения $\mathrm{P}=20$ Вт, частота излучения $f=20-100$ кГц, длительность лазерного импульса $\tau=5 \ldots 200 \mathrm{нc}$, диаметр пучка лазера в зоне фокуса $d_{n}=35$ мкм. 
Таблица 2

Параметры для расчета

\begin{tabular}{|c|c|c|c|}
\hline № & $\begin{array}{l}\text { Пара- } \\
\text { метр }\end{array}$ & Название & Метод определения \\
\hline 1 & $P$ & $\begin{array}{c}\text { Среднее значение мощности } \\
\text { лазера }\end{array}$ & Определяется экспериментально \\
\hline 2 & $f$ & Частота лазерного излучения & $\begin{array}{c}\text { Варьируется посредством устройства управления } \\
\text { в диапазоне, указанным производителем }\end{array}$ \\
\hline 3 & $\tau$ & $\begin{array}{c}\text { Длительность лазерного } \\
\text { импульса }\end{array}$ & $\begin{array}{c}\text { Варьируется посредством устройства управления } \\
\text { в диапазоне, указанным производителем }\end{array}$ \\
\hline 4 & $d_{\Pi}$ & $\begin{array}{l}\text { Диаметр пятна лазера } \\
\text { в плоскости обработки }\end{array}$ & $\begin{array}{c}\text { Указывается производителем, для лучшей точности } \\
\text { допускается определять экспериментально }\end{array}$ \\
\hline 5 & $R$ & $\begin{array}{c}\text { Коэффициент отражения } \\
\text { обрабатываемой поверхности }\end{array}$ & $\begin{array}{c}\text { Табличное значение для каждого материала, для лучшей } \\
\text { точности допускается определять экспериментально }\end{array}$ \\
\hline 6 & $\alpha$ & Показатель поглощения & $\begin{array}{c}\text { Определяется экспериментально для конкретного } \\
\text { материала }\end{array}$ \\
\hline
\end{tabular}

Для определения средней мощности лазерного излучения использовался измеритель РМ 200 с датчиком S314.

При измерении мощности лазерного излучения варьировались параметры длительности импульса $\tau$ и частоты лазерного излучения $f$. Исследования проводились для двух режимов работы лазера с мощностью $P=16,7 \mathrm{Bт} \mathrm{(} \tau=200$ нс и $f=20$ кГц) и $P=8,17$ Вт $(\tau=200$ нс и $f=20$ кГц $)$.

Согласно [8] коэффициент отражения медной поверхности $\mathrm{R}$ равен 0,91 . Однако для более точных расчетов были проведены дополнительные измерения на лабораторных образцах из меди.

Измерение коэффициента отражения проводилось на специальном стенде (рисунок 1), состоящем из сферы Labsphere (1) и осветителя Koehler Illuminator KI-120 (2).

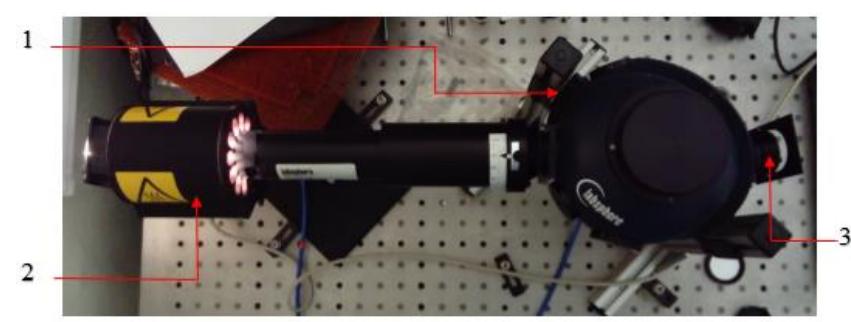

Рис. 1. Стенд для измерения коэффициента отражения

1 - сфера, 2 - осветитель, 3 - место расположения исследуемых образцов

Лабораторный образец из меди располагался на внутренней поверхности сферы. Осветитель осуществлял подсветку лабораторного образца сфокусированным пучком света, диаметром 12 мм с заданной интенсивностью $I_{0}$. Сфера регистрировала интенсивность полного отражения

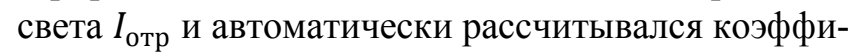

циент отражения как отношение интенсивности отраженного излучения к падающему $\frac{I_{\text {отр }}}{I_{0}}$. По результатам десяти измерений было получено среднее значение коэффициента отражения $R=0,56$.

Последним искомым параметром является показатель поглощения $\alpha$. Данный параметр характеризует ослабление интенсивности излучения заданной длины волны в материале. Для металлов, облучаемых лазерным излучением с длиной волны 1064 нм в открытых источниках [4], [5] приведены приближенные значения $10^{5} \ldots 10^{6} 1 / \mathrm{M}$.

Его нахождение возможно посредством проведения эксперимента с подачей одиночного лазерного импульса длительностью $\tau=200$ нс, длиной волны 1064 нм на поверхность пластинки из меди. Согласно формуле (7), пороговая интенсивность излучения необходимая для испарения меди, с учетом измеренных значений коэффициента отражения $\mathrm{R}=0,56$ составляет $I_{\text {имп }}^{\text {пор }}=\frac{8,263 \cdot 10^{7}}{(1-R) \sqrt{\tau}}=$ $\frac{8,263 \cdot 10^{7}}{(1-0,56) \sqrt{200 \cdot 10^{-9}}}=4,199 \cdot 10^{11} \mathrm{BT} / \mathrm{M}^{2}$. Если подставить полученное значение в закон Бугера (5), то получим следующее соотношение:

$$
4,199 \cdot 10^{11}=\frac{4 \cdot P}{f \cdot \tau \cdot \pi \cdot d_{\Pi}^{2}}(1-R) e^{-\alpha x}
$$

После подстановки параметров $P, f, \tau, d_{\text {п }}$, $R$, входящих в состав соотношения (9), которые были определены ранее, получим следующий результат:

$$
\alpha=-\frac{\ln 0,22}{x}
$$

Физический смысл переменной $x$ в (10)глубина лунки, полученной при испарении материала одним лазерным импульсом. Для определения $x$ был проведен эксперимент. Пластинка из меди была расположена на фокусном расстоянии от ска- 
наторной головки лазерного комплекса. На пластинку был подан одиночный лазерный импульс, длительностью 200 нс. Исследование геометрических размеров лунки, полученной при испарении материала осуществлялось на профилометре Sensofar SNeox.

На рис. 2 показана фотография результатов измерения глубины лунки на профилометре.
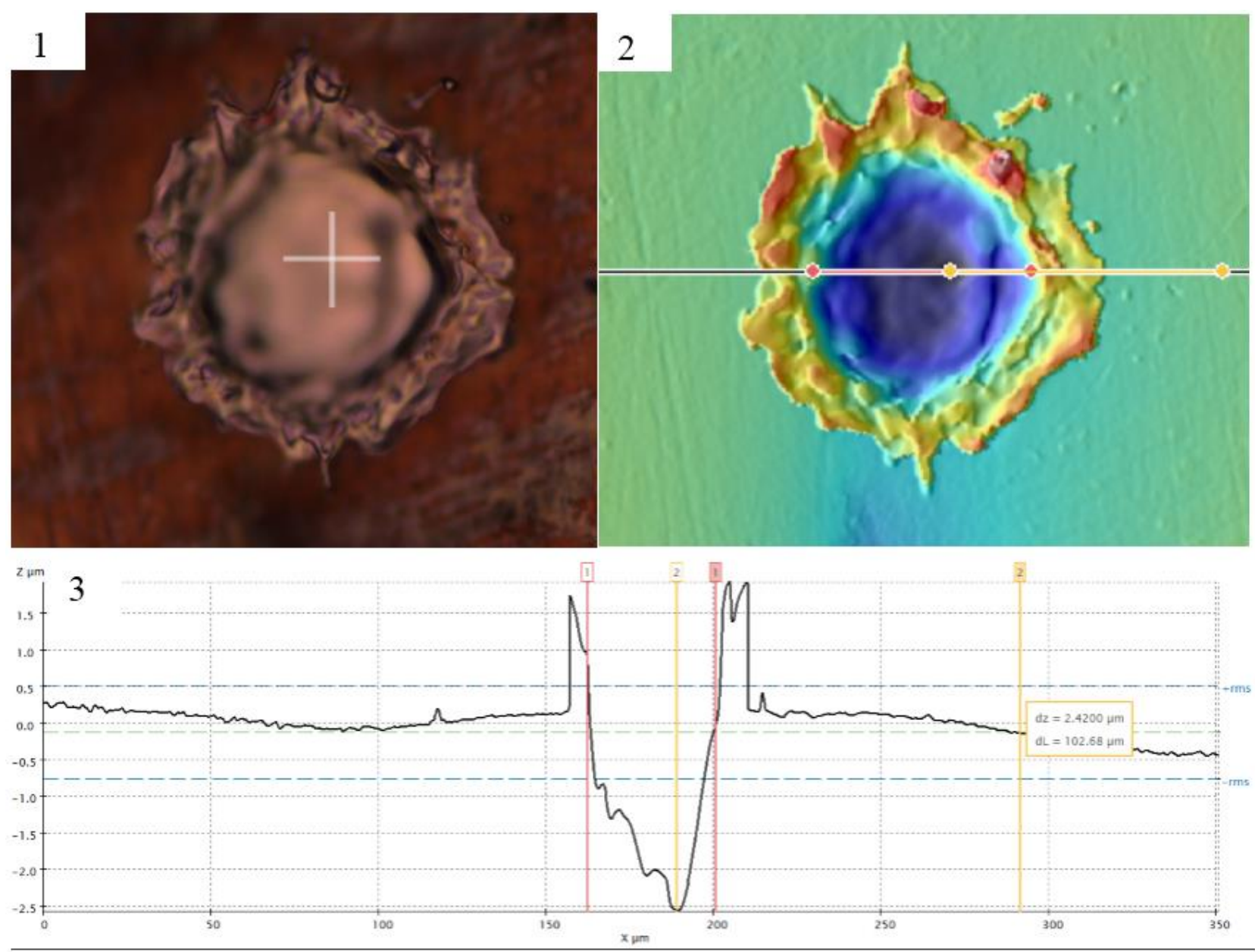

Рис. 2. Фотография результатов измерения глубины лунки на профилометре

1 - фотография полученной лунки через объектив профилометра, 2 - фотография 3D поверхности лунки, полученная посредством измерения, 3 - фотография профиля лунки

По результатам измерения, глубина лунки $x$ составила 2,42 мкм. При подстановке полученного значения в формулу (10) получим $\alpha=-\frac{\ln 0,22}{x}=6,257 \cdot 10^{5} 1 / \mathrm{M}$.

Расчет глубины лунок при воздействии одиночных импульсов на мишень из меди проводился по формуле (8) $x=-\frac{\ln \frac{2,07 \cdot 10^{7} \cdot f \cdot \sqrt{\tau} \cdot \pi \cdot d_{\Pi}^{2}}{P \cdot(1-R)^{2}}}{\alpha}$.

Для сравнения, расчет проводился также по указанной ранее формуле (1), которая была предложена в работе [4] и с учетом (3), (4) имела следующий вид: $h=\frac{P(1-R)}{f \frac{\pi \cdot d_{\Pi}^{2}}{4} L_{и}}$.

Исходные данные для расчета по формуле 8 приведены в табл. 3.
Таблица 3

Параметры для расчета для двух мощностей лазерного излучения

\begin{tabular}{|c|c|c|c|}
\hline № & Параметр & $\begin{array}{c}\text { Значение, } \\
\text { вариант 1 }\end{array}$ & $\begin{array}{c}\text { Значение, } \\
\text { вариант 2 }\end{array}$ \\
\hline 1 & $P$ & $16,70 \mathrm{Bт}$ & $8,17 \mathrm{Bт}$ \\
\hline 2 & $f$ & 20 кГц & 20 кГц \\
\hline 3 & $\tau$ & $200 \mathrm{Hc}$ & $100 \mathrm{Hс}$ \\
\hline 4 & $d_{\Pi}$ & 35 мкм & 35 мкм \\
\hline 5 & $R$ & 0,56 & 0,56 \\
\hline 6 & $\alpha$ & $6,257 \cdot 10^{5}$ & $6,257 \cdot 10^{5}$ \\
\hline
\end{tabular}

С целью определения сходимости полученных результатов посредством комплекса лазерной обработки было нанесено 2 варианта поверхностной микроструктуры, соответствующей двум вариантам проведенного расчета. На рис. 3 приведены 
изображения микроструктур, полученные с помощью измерения профилометром. Результаты измерения глубины 20 лунок микроструктуры на профилометре позволили определить среднее значение глубины лунок с учетом абсолютной погрешности:

$$
\text { - } x_{1}=2,258 \pm 0,632(P=0,95) \text { для микро- }
$$

структуры варианта 1 ,

$$
\text { - } x_{2}=1,771 \pm 0,571(P=0,95) \text { для микро- }
$$

структуры варианта 2.

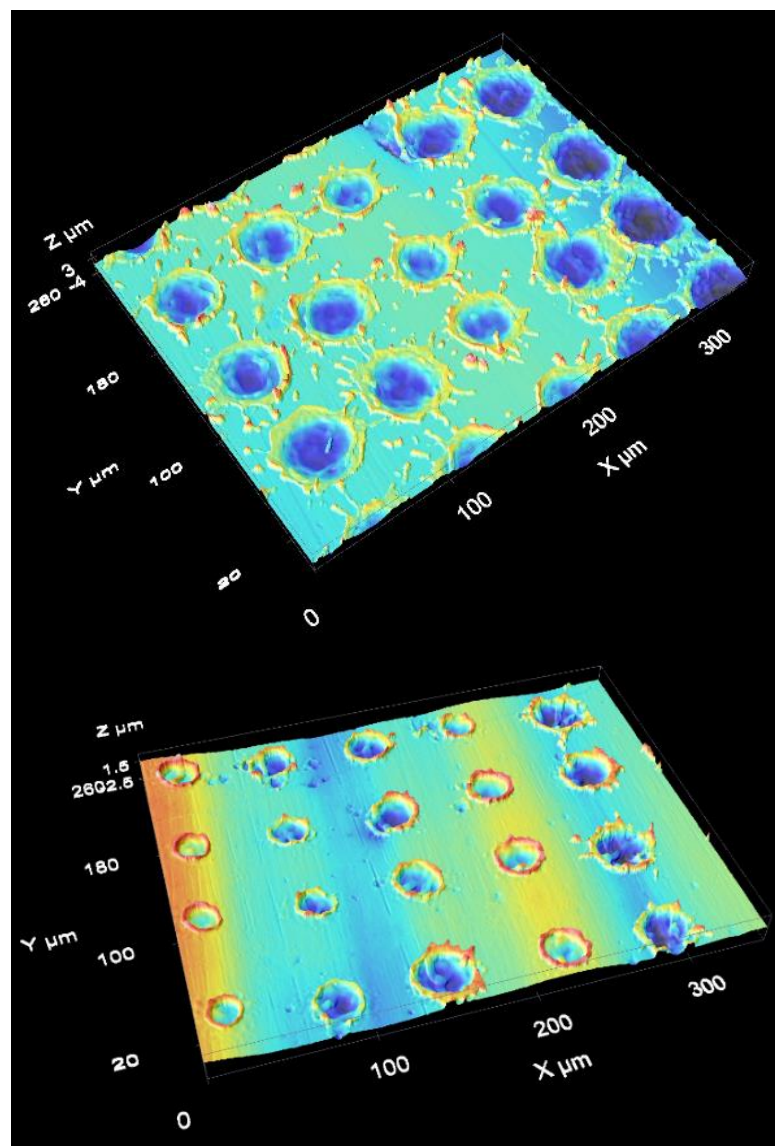

Рис. 3. Фотографии микроструктуры варианта «1» и варианта «2», полученные на профилометре

$$
\text { ЛИ Т ЕР А Т У Р А }
$$

1. Разработка методики совершенствования рабочих трехмерных поверхностей лезвий коньков с учетом особенностей соревновательных характеристик бега в шорттреке: отчет о НИР. рук. Кулагин Ю.А. Исполн: Аватинян Г.А., Баранов Е.О., Шинкарев А.С. и др. ФГУП «ЦНИИХМ». 2014. № НИОКТР 114111870038.

2. Аватинян Г.А., Баранов Е.О., Кулагин Ю.А. Лазерное структурирование поверхности пуль. 13-я Всероссийская научная конференция «Технологии и материалы для экстремальных условий». Звенигород. 2018.

3. Аватинян Г.А., Баранов Е.О., Кулагин Ю.А. и др. Лазерная модификация поверхностей подшипников пикосекундным лазером. 12-я Всероссийская научная конференция «Технологии и материалы для экстремальных условий. Туапсе. 2017.
Сравнение расчетных и измеренных значений глубины профиля микроструктуры приведены в табл. 4.

\section{ВЫВОДЫ}

Использование закона Бугера-ЛамбертаБера позволяет рассчитывать глубину лунок поверхностной микроструктуры при облучении единичным наносекундным лазерным импульсом с более высокой точностью чем существующее эмпирическое соотношение благодаря учету большего количества оптических и теплофизических параметров лазера и мишени.

Точность расчетов также обеспечивается возможностью экспериментального измерения средней мощности лазера и коэффициента отражения медного образца. В случае проведенных экспериментов, измеренное значение коэффициента отражения меди отличалось от табличного на $38,5 \%$.

Закон Бугера-Ламберта-Бера рекомендуются использовать в задачах по микрообработке поверхности, когда важен контроль геометрических размеров профиля микроструктуры.

Таблица 4

Сравнение расчетных и измеренных значений глубины профиля микроструктуры

\begin{tabular}{|c|c|c|}
\hline Показатели & Вариант 1 & Вариант 2 \\
\hline $\begin{array}{c}\text { Расчетное значение } \\
\text { глубины (h), мкм }\end{array}$ & 7,965 & 3,897 \\
\hline $\begin{array}{c}\text { Расчетное значение } \\
\text { глубины (х), } \\
\text { по Бугеру, мкм }\end{array}$ & 2,417 & 1,828 \\
\hline Измеренное (х), мкм & $\begin{array}{c}2,258 \pm 0,632 \\
\mathrm{P}=0,95\end{array}$ & $\begin{array}{c}1,771 \pm 0,571 \\
\mathrm{P}=0,95\end{array}$ \\
\hline Сходимость (h), \% & 71,7 & 54,6 \\
\hline $\begin{array}{c}\text { Сходимость (x) } \\
\text { по Бугеру \% }\end{array}$ & 93,8 & 96,9 \\
\hline
\end{tabular}

\section{REFERENCES}

1. Development of a methodology for improving the working three-dimensional surfaces of the blades of skates taking into account the features of the competitive characteristics of running in short track: research report // hands. Kulagin Yu.A. Performed by: Avatinyan G.A., Baranov E.O., Shinkarev A.S. et al. // FSUE "TsNIIHM". 2014. NIOKTR 114111870038 .

2. Avatinyan G.A., Baranov E.O., Kulagin Yu.A. Laser structuring of the surface of bullets. 13th All-Russian scientific conference "Technologies and materials for extreme conditions". Zvenigorod, 2018.

3. Avatinyan G.A., Baranov E.O., Kulagin Yu.A. et al. Laser modification of bearing surfaces with a picosecond laser. 12th All-Russian scientific conference "Technologies and materials for extreme conditions. Tuapse. 2017. 
4. Парфенов В.A. Лазерная микрообработка материалов. СПб.: СПбГЭТУ «ЛЭТИ». 2011.

5. Вейко В.П. Опорный конспект лекций по курсу «Физикотехнические основы лазерных технологий». СПб. СПбГУ ИТМО. 2011.

6. ГОСТ 25786-83 Лазеры. Методы измерений средней мощности, средней мощности импульса, относительной нестабильности средней мощности лазерного излучения. М.: Издательство стандартов. 1983.

7. LDesigner F3: https:// ateko.ru/product/oborudovanie/ldesigner-f-seriya/ldesigner-f3/ (дата обращения 2021-08-08).

8. Звелто О. Принципы лазеров. - М.: Мир. 1990. 719 с.
4. Parfenov V.A. Laser micromachining of materials. SPb. SPbGETU "LETI". 2011.

5. Veiko V.P. Basic lecture notes for the course "Physico-technical foundations of laser technologies". SPb.: SPbSU ITMO. 2011.

6. GOST 25786-83 Lasers. Methods for measuring average power, average pulse power, relative instability of average power of laser radiation. M .: Publishing house of standards. 1983.

7. LDesigner F3: https:// ateko.ru/product/oborudovanie/ldesigner-f-seriya/ldesigner-f3/ (date of the application 202108-08).

8. Zvelto O. Principles of lasers. M .: Mir, 1990. 719 p.

Поступила в редакииюо (Received) 01.08.2021 Принята к опубликованию (Accepted) 03.09.2021 